\title{
REVIEW ARTICLE Experimental models assessing bilirubin neurotoxicity
}

\author{
Giulia Bortolussi ${ }^{1}$ and Andrés F. Muro ${ }^{1}$
}

The molecular and cellular events leading to bilirubin-induced neurotoxicity, the mechanisms regulating liver and intestine expression in neonates, and alternative pathways of bilirubin catabolism remain incompletely defined. To answer these questions, researchers have developed a number of model systems to closely recapitulate the main characteristics of the disease, ranging from tissue cultures to engineered mouse models. In the present review we describe in vitro, ex vivo, and in vivo models developed to study bilirubin metabolism and neurotoxicity, with a special focus on the use of engineered animal models. In addition, we discussed the most recent studies related to potential therapeutic approaches to treat neonatal hyperbilirubinemia, ranging from anti-inflammatory drugs, activation of nuclear receptor pathways, blockade of bilirubin catabolism, and stimulation of alternative bilirubin-disposal pathways.

Pediatric Research (2020) 87:17-25; https://doi.org/10.1038/s41390-019-0570-x

\section{INTRODUCTION TO BILIRUBIN METABOLISM AND ITS IMPAIRMENT}

Unconjugated hyperbilirubinemia is caused by the alteration of bilirubin metabolism. Unconjugated bilirubin (UCB) is poorly soluble in body fluids and, once produced, it binds to serum albumin, reaches the liver, entering the hepatocytes by passive diffusion or active transport. In the liver, ${ }^{1}$ UDP glucuronidase transferase $1 \mathrm{~A} 1$ (UGT1A1) converts UCB into mono- and di-glucuronidated bilirubin, which are secreted into the bile fluid via the multidrug resistance protein 2 (ABCC2), then reaching the intestine. ${ }^{2}$

Neonatal jaundice occurs in $60 \%$ of term neonates and almost all pre-term newborns, ${ }^{3,4}$ and is often considered beneficial due to the antioxidant properties of bilirubin. ${ }^{5-7}$ It is caused by a transient delay in UGT1A1 gene activation that self-resolves within few days. ${ }^{8}$ However, during severe unconjugated hyperbilirubinemia (SUH) the excess of UCB that cannot bind to albumin [also called free bilirubin (Bf)] accumulates in lipid-rich tissues, such as the brain, reaching toxic levels and causing neurological damage and, potentially, death by kernicterus. ${ }^{9}$

$\mathrm{SUH}$ in neonates is caused by genetic and non-genetic factors, or by a combination of both., ${ }^{9,10}$ Several non-genetic factors contribute to SUH, ranging from hypoxia and infections to hepatic disorders, neonatal jaundice, and breast milk jaundice. ${ }^{10}$

Breast milk jaundice has been recognized as an important factor for the development of SUH, since it acts both as a trigger and as a concomitant effector. Although it was described as early as $1964,{ }^{11}$ only recent studies showed that suppression of intestinal UGT1A1 expression by breast milk feeding is associated with higher UCB levels (see ref. ${ }^{12}$ and the "Hepatic and gastrointestinal factors contributing to neonatal hyperbilirubinemia" Section below for a detailed description).

Management of neonatal hyperbilirubinemia includes guidelines for standard and effective treatments to reduce bilirubin toxicity. ${ }^{3}$ Among them, intensive phototherapy (PT) is the universal treatment for every type of unconjugated hyperbilirubinemia. ${ }^{13}$
However, some neonates fail to respond to intensive PT due to concurrent factors and may need more invasive procedures. ${ }^{3,14-20}$ Thus, further efforts are required to dissect the mechanisms leading to bilirubin-induced neurological damage. A number of in vitro and in vivo models have been developed in order to study different aspects of bilirubin neurotoxicity and to test novel experimental treatments aiming at limiting the risk of neurological damage, and are described below.

\section{IN VITRO AND IN VIVO MODELS TO STUDY BILIRUBIN METABOLISM, TOXICITY, AND POTENTIAL THERAPEUTIC APPROACHES}

More effective treatments and biomarkers to trace disease progression will be developed after a deeper understanding of the key mechanism underneath. To reach these goals it is essential to generate and study model systems that closely recapitulate the main characteristics of the disease of interest. In the next sections, we will describe the valuable models studied so far to understand the pathophysiology of SUH and discuss their important impact on the development of new therapeutic strategies to tackle the disease.

In vitro models

The exposure of cell types of different origins to UCB allowed the exploration of several molecular aspects of bilirubin toxicity, ranging from oxidative stress, DNA damage, endoplasmic reticulum (ER) stress, bilirubin-clearance mechanisms by transporters, and autophagy. ${ }^{21-29}$

Among the in vitro cell cultures extensively used to study bilirubin neurotoxic, we list two major groups: immortalized cell lines and primary cultures. To the former group belong human neuroblastoma cell lines (SH-SY5Y), ${ }^{21,22,25,30}$ HeLa cells (cervical cancer) ${ }^{23,24,31}$ Hepa 1c1c7 mouse hepatoma cell line, 26,32,33 and human U87 astrocytoma cells. ${ }^{22,34}$ HeLa cells have also been modified to stably express a reporter gene, such as in the case of HeLa DR-GFP cells. ${ }^{23}$

\footnotetext{
${ }^{1}$ International Centre for Genetic Engineering and Biotechnology (ICGEB), Padriciano, 99, 34149 Trieste, Italy

Correspondence: Andrés F. Muro (muro@icgeb.org)
}

Received: 17 May 2019 Revised: 29 July 2019 Accepted: 16 August 2019

Published online: 7 September 2019 
These cell types exposed to different concentrations of UCB allowed the exploration of several molecular aspects of bilirubin toxicity. ${ }^{21-26}$

Key information was also provided by experiments performed in rat and mouse primary cultures of neurons, astrocytes, microglia, oligodendrocytes, endothelia cells (see ref. ${ }^{35}$ for a detailed description), and embryonic fibroblasts, ${ }^{27,36}$ systems having properties closer to their original in vivo situation, without carrying the complexity of the whole tissue. However, the loss of natural environment is accompanied by a change in the cell-cell relationships, and morphological and physiological properties.

Among the neuronal cells, primary hippocampal neurons are the main cell type used to test bilirubin response. These cells exposed to bilirubin show important morphological, biochemical/metabolic, and molecular changes reflecting in vivo features, such as reduction in axon and dendrites, increased cell death, increased oxidation and mitochondrial dysfunction, and overexpression of protection mechanisms (active transporters, detoxification pathways, prosurvival mechanism, and inflammatory markers). ${ }^{35}$ In addition, less differentiated neuronal cell types appeared to be more sensitive to bilirubin compared to differentiated/aged ones. ${ }^{37}$

Reports both from patients affected by kernicterus and studies in animal models showed that bilirubin toxicity is region-specific, affecting only selected areas of the brain and specific neurons in these regions. ${ }^{9,38-40}$ The causes might be related to several factors, ranging from bilirubin regional load, capacity of the specific neuronal cell types to clear bilirubin from the tissue, to cell type, and the level of differentiation/aging. ${ }^{36,38,41-43}$

Oligodendrocytes are very susceptible to the bilirubin insult mirroring, to some extent, the observations reported in neurons. In addition, oligodendrocytes showed reduced myelin basic protein production with the consequent impairment of myelin sheet formation and neuronal axonal function. ${ }^{44}$

Glial cells (astrocytes and microglia) respond promptly to bilirubin by triggering mitogen-activated protein kinase pathways (p38, JNK1/2, and ERK1/2) and by releasing pro-inflammatory mediators (tumor necrosis factor- $\alpha$ (TNFa), interleukin-1 $\beta$ (IL-1 $1 \beta$ ), IL6 , nuclear factor- $\mathrm{KB}$ (NF-kB); see ref. ${ }^{35}$ for a detailed description).

A higher level of complexity was reached with heterotypic cell cultures (also named co-cultures), since this system allowed the exploration of the crosstalk between different neuronal cell types in response to UCB. A pivotal study showed that astrocytes protected neurons from UCB toxicity when simultaneously exposed to bilirubin, while they were detrimental for neuronal survival when extensively stimulated. ${ }^{45}$

However, these approaches have some disadvantages consequent to the loss of the natural environment, with changes in cell-cell relationships, morphological and physiological properties, potential contamination with different population of cells, and their limited lifespan in culture.

Ex vivo models: organotypic cultures

Researchers developed more sophisticated assays based on slices of the entire brain (or a region of interest), namely organotypic cultures (or slices), being ex vivo models in which an isolated tissue is cultured in vitro. Culturing brain slices couples the benefits of in vivo studies to those of in vitro cultures, allowing the study of selected brain regions at the desired developmental stage. They transiently maintain the structure, cellular complexity, and heterogeneity of the organ and, in addition, can be exposed to different culture conditions.

Very few studies exploited organotypic brain cultures to study bilirubin toxicity, ${ }^{43,46-48}$ in particular hippocampal slices, showing the impairment of synaptic plasticity by bilirubin and the involvement of microglia as a key player in UCB-induced neurotoxicity. ${ }^{46,47}$ Likewise, researchers tested different types of drugs for their ability to thwart cytotoxicity when used in combination with bilirubin, such as glycoursodeoxycholic acid, minocycline, curcumin, and indomethacin. ${ }^{43,46}$
Interestingly, Silva et al. ${ }^{49}$ evaluated bilirubin toxicity in nondepleted and microglia-depleted hippocampal organotypic slices showing that bilirubin-induced cell death increased in non-depleted slices, indicating that microglia can exacerbate bilirubin toxicity.

Some results need further exploration, as those from Dal Ben et al., ${ }^{43}$ who showed that organotypic slices derived from hippocampus are more susceptible to bilirubin than the inferior and superior colliculus or cerebellum, or those of Dani et al., ${ }^{48}$ who showed that more immature slices were more resistant to bilirubin, ${ }^{48}$ being the opposite of what was observed in in vivo and other in vitro models of hereditary unconjugated hyperbilirubinemia. ${ }^{37,39,50-56}$ Such discrepancies are most likely due to different experimental conditions applied, such as developmental stages of the donor brains that might influence neuronal susceptibility, days in culture prior to use, concentrations of bilirubin applied, which can greatly differ from the one observed in vivo, as well as bilirubin-albumin ratio or Bf calculations in the culturing media compared to in vivo.

\section{In vivo models}

As in the case of in vitro assays, isolated tissues do not completely replicate all disease features manifested in patients. This is accomplished to a good extent by the use of animal models.

In the following sections we will discuss in detail in vivo models and their important contribution to the understanding of bilirubin toxicity (Table 1).

Genetic in vivo models of severe hyperbilirubinemia. Naturally occurring mutations: the Gunn rat. It was described in 1938 as a spontaneously jaundiced mutant strain of Wistar rats, ${ }^{57,58}$ and the vast majority of in vivo knowledge on bilirubin metabolism and toxicity derives from the study of this model. Hyperbilirubinemia in these animals is caused by the complete deficiency of Ugt1a1 enzymatic activity, consequent to the presence of a premature stop codon in exon 4 of the Ugt1a gene, resulting in a truncated protein $^{59}$ that is rapidly degraded. ${ }^{60}$ Bilirubin affects brain development, with cerebellar hypoplasia and alterations of brainstem auditory-evoked potentials (BAEPs). ${ }^{55,56,61}$ However, untreated Gunn rats only partially recapitulate the human syndrome, reaching adulthood and being fertile. Thus, Gunn rats are frequently treated with different hemolytic drugs or albumin-bilirubin displacers to exacerbate hyperbilirubinemia (i.e., phenylhydrazine (PHZ), sulfadimethoxine; see the "Pharmacologically induced in vivo models" Section below for details).

Induced mutations. The development of methods for the manipulation of the mouse genome have boosted the exploration of key aspects of the disease, by generating constitutive and conditional knockout, knock-in, and transgenic strains. ${ }^{12,36,39,50,51,62-64}$ These new animal models provided an extraordinary contribution to the understanding of severe neonatal hyperbilirubinemia.

The first engineered mouse model of SUH was generated by disrupting Ugt 1 exon 4 by the neomycin cassette. ${ }^{64}$ Mutant mice do not express Ugt1a1, present neonatal hyperbilirubinemia and lethality within 11 days after birth, and are also a model of the Crigler-Najjar syndrome type I. $^{64}$ Since these animals die soon after birth, to study neonatal hyperbilirubinemia the same group generated other two strains by introducing the UGT1A*1 WT and UGT1A $1 * 28$ Gilbert human alleles in the Ugt1a-null background. ${ }^{63}$ The humanized $\mathrm{Tg}\left(\mathrm{Ugt1}^{\mathrm{A}^{* 1}}\right) \mathrm{Ugt} 1^{-/-}$strain rescued neonatal lethality. However, bilirubin levels in the humanized Gilbert mice were higher, and, interestingly, $10 \%$ of $\mathrm{Tg}\left(\mathrm{Ugt}^{\mathrm{A} 1^{*} 28}\right) \mathrm{Ugt} 1^{-/-}$mice developed extremely high bilirubin levels about 2 weeks after birth and died from bilirubin toxicity showing seizures and dystonia. The $\operatorname{Tg}\left(\mathrm{Ugt}^{\mathrm{A} 1^{*} 28}\right) \mathrm{Ugt}^{-/-}$mouse model was further used to study other aspects of bilirubin metabolism, by mating them with pregnane $X$ receptor (PXR) and constitutive active receptor (CAR)-null strains, ${ }^{12,52,62,63,65-67}$ as detailed in the 
Table 1. Animal models for the study of bilirubin neurotoxicity and metabolism

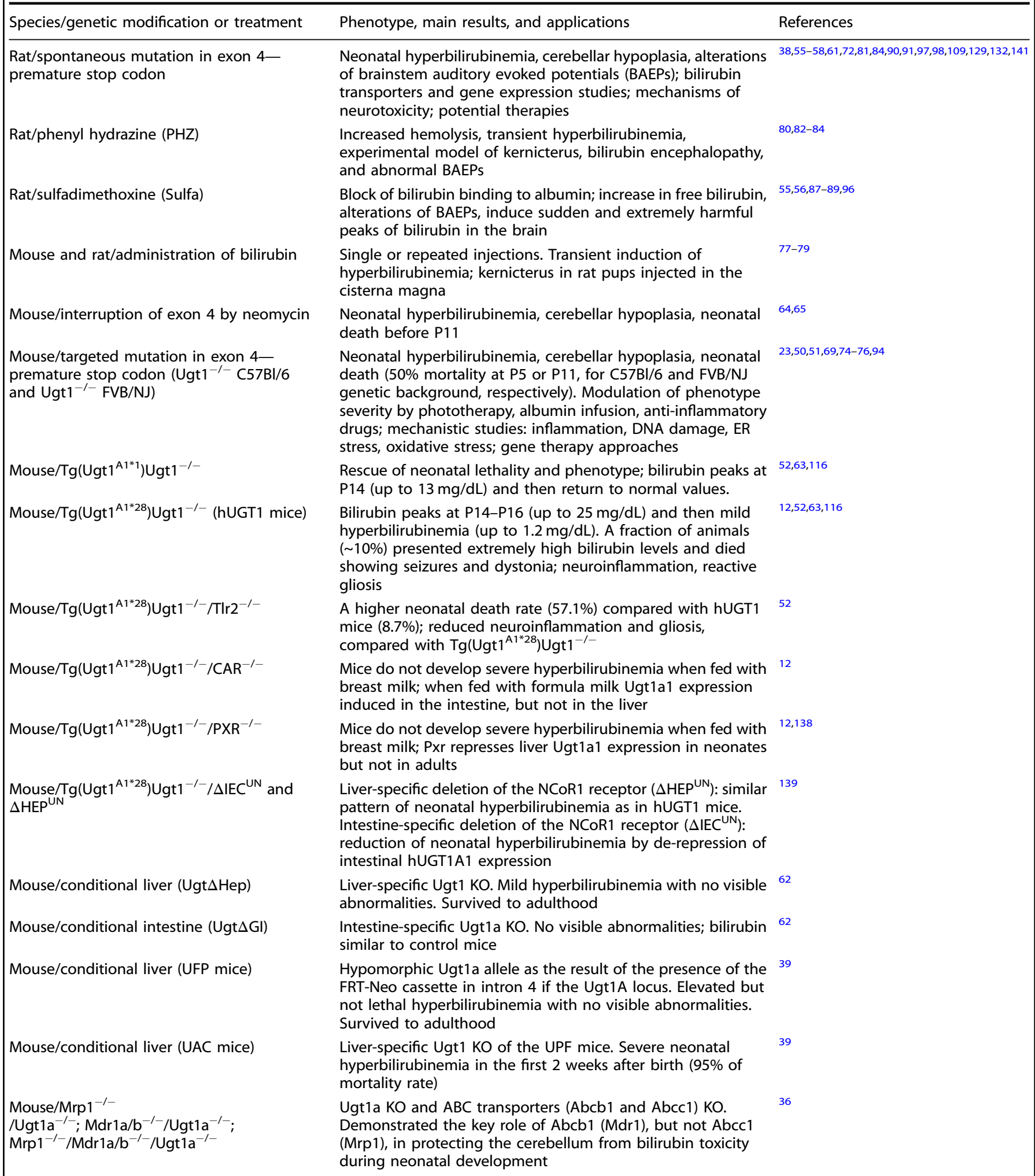

"Hepatic and gastrointestinal factors contributing to neonatal hyperbilirubinemia" Section below.

Since only a small and unpredictable fraction of $\mathrm{Tg}\left(\mathrm{Ugt}_{1}{ }^{\mathrm{A} 1 * 28}\right)$ Ugt ${ }^{-1-}$ developed kernicterus, a liver-specific knockout (KO) of Ugt1a1 gene was generated. ${ }^{39,62}$ These animals develop severe neonatal hyperbilirubinemia in the first 2 weeks after birth $(95 \%$ of mortality rate), recapitulating more closely the human syndrome. $^{39}$ Likewise, to study the contribution of the liver and intestine in the early postnatal days, a mouse strain with intestinespecific inactivation of the Ugt1a gene was also generated. ${ }^{62}$

In parallel, our laboratory generated a mouse strain containing a one-base deletion in the common exon 4 of the Ugt1 locus, 
identical to the one present in the Gunn rat, resulting in the absence of Ugt1a1 protein expression and bilirubin glucuronidation activity in the liver of these mice. ${ }^{51}$ The genetic mutation was transferred to two genetic backgrounds (C57BI/6 and FVB/NJ), recapitulating the major features of severe neonatal unconjugated hyperbilirubinemia, with peaks of bilirubin neurotoxicity, cerebellar abnormalities, and mortality at different postnatal periods $(50 \%$ mortality at postnatal day 5 (P5) and P11, respectively; refs. ${ }^{50,51}$ ). This mouse model, together with the Gunn rat, is also used to develop gene therapy approaches for Crigler-Najjar syndrome type I..$^{1,68-73}$

Ugt1a-null mouse models present a stronger phenotype than Gunn rats, with more severe neurological damage and ultimately death. ${ }^{5,51,64}$ The reasons for the phenotype differences between Gunn rats and mutant mouse strains, and between the two mouse strains, are not yet clear, but suggest that susceptibility to bilirubin neurotoxicity and survival are strain and species specific. ${ }^{50}$

These strains were used to study different aspects of the mechanisms of bilirubin neurotoxicity, therapeutic approaches, and the role of modifier genes of the $A B C$ family of transporters (see section "The use of experimental animal models to study the mechanisms of bilirubin neurotoxicity" below). ${ }^{23,36,50,51,68,70,74-76}$

Pharmacologically induced in vivo models. In the absence of a genetic defect, the most straightforward approach to increase total bilirubin is to directly administer UCB to the animal. This procedure was successfully applied to neonatal rat or mouse pups, by single $\mathrm{e}^{77}$ or repeated intraperitoneal injections of bilirubin. ${ }^{78}$ Likewise, a kernicterus model in newborn rats was established by performing intra cisterna magna injection in 5-day-old rat pups. ${ }^{79}$ Conversely, hyperbilirubinemia was transiently induced in the brain of adult animals by intraventricular injection. ${ }^{78}$ The convenience of these methods resides in their simplicity, being at the reach of every laboratory. They allow the exploration of punctual spikes of bilirubin (in dose and time), offering a good estimation of dose vs. brain damage effect. However, these experimentally induced conditions only partially mimic the natural course of the disease.

The administration of $\mathrm{PHZ}$, a potent inducer of acute hemolytic anemia, leads to hyperbilirubinemia by increasing erythrocyte turnover (hemolysis). Spontaneously jaundiced (jj) Gunn rats treated with $\mathrm{PHZ}$ served to generate a more clinically relevant experimental model of kernicterus, which presented bilirubin encephalopathy and abnormal BAEPs, comparable to human hyperbilirubinemic newborns. ${ }^{80}$ This method was applied to adult WT Wistar rats and Swiss mice showing the upregulation of the Abcc1 transporter in the liver and spleen by bilirubin, ${ }^{81}$ and the antioxidant properties of a Phyllanthus amarus root, ${ }^{82}$ respectively. More recently, $\mathrm{PHZ}$ administration was used in Gunn rats to test the efficacy of Human serum albumin administration and exchange transfusion in combination with bilirubin albumin displacers. ${ }^{83,84}$

Finally, the most used method to induce hyperbilirubinemia in vivo is the administration of sulfadimethoxine, a displacer of bilirubin from albumin binding sites. ${ }^{85-87}$ Sulfadimethoxine administration increases the fraction of $\mathrm{Bf}$, which mobilizes to lipophilic tissues such as the brain, and is accompanied by a drop of systemic bilirubin. This treatment was exploited both in neonatal and adult Gunn rats to induce sudden and extremely harmful peaks of bilirubin in the brain, ${ }^{87}$ allowing the exploration of several aspects of bilirubin toxicity, ${ }^{38,55,88-90}$ also in combination with other treatments. ${ }^{83,84}$

THE USE OF EXPERIMENTAL ANIMAL MODELS TO STUDY THE MECHANISMS OF BILIRUBIN NEUROTOXICITY

Modulation of bilirubin neurotoxicity by PT

Severity of the phenotype in Ugt $1^{-1-}$ mice is associated with the genetic background. ${ }^{50}$ Disease phenotype in $\mathrm{Ugt}^{-/-} \mathrm{C} 57 \mathrm{BI} / 6$ mice is very severe, with death of $50 \%$ of pups at $\mathrm{P} 5$ and more severe brain damage compared to Ugt $1^{-/-} \mathrm{FVB} / \mathrm{NJ}$ animals (death of $50 \%$ at $\mathrm{P} 11$ ), suggesting that other factors (i.e., modifier genes) are associated to bilirubin neurotoxicity. These two strains respond differently to PT treatment, since Ugt $1^{-1-} \mathrm{C} 57 \mathrm{BI} / 6$ mice succumb despite $12 \mathrm{~h}$ /day PT, while Ugt $1^{-1-}$ FVB/NJ animals survive, fully rescuing the phenotype. Importantly, the neurotoxic effects of bilirubin can be modulated by the PT treatment duration and developmental stage of the cerebellum. ${ }^{50}$

Clear differences between Ugt1 ${ }^{-1-}$ FVB/NJ animals and Gunn rats are observed: the most critical period for survival of Ugt $1^{-1-}$ FVB/NJ mice is between P8 and P12, and 15 days of PT are necessary to rescue lethality. ${ }^{50}$ On the contrary, in Gunn rats a single 24-h dose of PT is enough to prevent hypoplasia in the cerebellum, with treatment at day 7 being most effective. ${ }^{56,91}$ Thus, the Ugt1 $1^{-/-} \mathrm{FVB} / \mathrm{NJ}$ mouse model is an excellent and versatile model to characterize the molecular mechanisms of bilirubin toxicity during the neonatal phase, a critical period of human development. ${ }^{50}$

Oxidative stress, DNA and RNA damage, and post-transcriptional modifications

Oxidative damage is widely recognized as a mechanism through which bilirubin causes cytotoxicity and cellular death (reviewed in refs. ${ }^{35,92}$ ) Elevated concentrations of bilirubin activate Nrf2mediated pathway both in vitro and in vivo. ${ }^{93,94}$ However, while in vitro cell cultures exposed to bilirubin activate multiple Nrf2-, P38-, and MEK1/2-mediated antioxidant response genes to neutralize toxicity, ${ }^{93}$ in vivo studies showed that oxidoreductase and antioxidant processes are downrepresented in the cerebellum of hyperbilirubinemic Ugt $1^{-1-} \mathrm{C} 57 \mathrm{BI} / 6$ mice. ${ }^{94}$ The levels of key players of oxidative stress defenses, such as Prdx2 and Prdx6 (and their reduced forms), SOD1, and DJ-1, are decreased compared to healthy littermates. In vivo, the P38 pathway is activated in response to severe neonatal hyperbilirubinemia in Purkinje cell layer. ${ }^{94}$ Elevated levels of $\mathrm{HO}-1$ and inflammation are present in $\mathrm{Tg}$ $\left(\right.$ Ugt $\left.1^{\mathrm{A} 1^{*} 28}\right) \mathrm{Ugt}^{-1-}$ and Ugt $1^{-1-}$ FVB/NJ animals, ${ }^{52,74}$ with HO-1 peaking in microglial cells in the latest phases of bilirubin neurotoxicity. ${ }^{74}$ Importantly, treatment with an anti-inflammatory agent reduces the response in a dose-dependent manner, partially rescuing lethality of Ugt $1^{-1-}$ animals. ${ }^{75}$ In contrast, treatment with a well-recognized antioxidant agent, such as $\mathrm{N}$-acetylcysteine (NAC), neither improves survival nor reduces neurological damage in Ugt $1^{-/-}$animals ${ }^{75}$ Likewise, HO-1 upregulation is abolished in $\operatorname{Tg}\left(\mathrm{Ugt}^{\mathrm{A}^{\mathrm{1}}{ }^{* 28}}\right) \mathrm{Ugt}^{-/-} / \mathrm{TIr}^{-/-}$mice, animals in which the TLR2dependent innate immunity is abolished. ${ }^{52}$

Induction of oxidative stress in the liver and intestine of humanized $\mathrm{Tg}\left(\mathrm{Ugt}^{\mathrm{A}^{* 1}}\right) \mathrm{Ugt} 1^{-/-}$mice resulted in the activation of phase II drug metabolism enzymes, such as Ugt1a1 and Cyp2b10, through the CAR pathway. ${ }^{95}$ This effect was lost in $\operatorname{Tg}\left(\mathrm{Ugt}^{\mathrm{A}^{* 1} 1}\right)$ Ugt ${ }^{-1} \mathrm{CAR}^{-1-}$, indicating a close connection between isothiocyanate-induced oxidative stress and hepatic but not intestinal CAR activation of detoxify response genes in vivo. Similar results were obtained when humanized mice were orally treated with NAC, preventing the phenethyl isothiocyanatemediated induction of hepatic Ugt1a1 and Cyp2b10. ${ }^{95}$

It has been shown that bilirubin-mediated oxidative stress affects cellular constituents such as DNA, RNA, and lipids. ${ }^{25,92,96,97}$ Recent findings revealed that bilirubin-induced DNA damage occurs both in vitro and in vivo in the cerebellum of Ugt $1^{-1-}$ FVB/ NJ mice, ${ }^{23}$ associated with the activation of homologous recombination and non-homologous end joining, the main DNA repair mechanisms.

On a related line of exploration, hyperbilirubinemia affects histone acetylation and DNA methylation in Gunn rat pups' cerebellum, modulating DNA accessibility, structure, and, thus, potentially affecting gene expression. ${ }^{98}$ Down-regulation of genes related to oligodendrocyte development and myelin sheets 
formation is observed, in line with the reduced myelination present in Ugt1a liver-specific KO mice ${ }^{39}$ and in a premature infant with kernicterus. $^{99}$

ER stress, inflammation, and autophagy

The first evidence that bilirubin induces ER stress response derives from transcriptome analysis of SH-SY5Y neuroblastoma cells. ${ }^{21}$ Likewise, studies using oligodendrocyte precursors and hepatoma cell lines showed that bilirubin triggers ER stress by increasing key factors such as GPR78, IRE1, ATF3, and CHOP. ${ }^{26,100}$

During the initial phases of ER stress response cells activate prosurvival mechanisms such as unfolded protein response. However, toxic levels of bilirubin inhibit proteasome degradation in neuronal cells both in vitro and in vivo, with the accumulation of unfolded proteins and activation of pro-apoptotic pathways. ${ }^{78}$ These events result in significant ER stress, ${ }^{101}$ as also evidenced in the cerebellum of the Ugt $1^{-1-} \mathrm{FV} / \mathrm{NJ}$ hyperbilirubinemic mouse model. $^{74}$

ER stress is an early event associated with bilirubin toxicity and neuronal cell death. In vitro, inhibition of CHOP or ER stress pathway increases cell survival, ${ }^{22}$ in line with in vivo studies in Ugt $1^{-1-}$ FVB/NJ mouse cerebellum, showing that key factors of the ER stress response (ATF3, Fas, and CHOP) are significantly stimulated at the initial stages of severe neonatal hyperbilirubinemia and persist thereafter. ${ }^{74}$ In particular, CHOP signal was localized in degenerating Purkinje neurons, supporting the concept that bilirubin-induced ER stress response is associated with apoptotic neurons. ${ }^{22,50,74,78}$

Neurodegeneration, increased oxidative stress, ER stress, and inflammation are intricately interconnected and, in turn, boost each other leading to unfavorable outcome. Numerous studies demonstrated the detrimental effect of bilirubin-mediated inflammation on neuronal cells, ${ }^{35}$ mediated by astrocytes ${ }^{45,102,103}$ and microglia activation ${ }^{49,104}$ through the release of proinflammatory mediators (IL-1 $\beta$, TNF- $\alpha$, NF-KB, IL-6, glutamate, and interferon- $\gamma$ ). Recent in vitro investigations connected ER stress response with inflammation through the activation of PERK and NF-K $\beta$ pathway in neuronal cells, but not in astrocytes. ${ }^{22}$ Inhibition of ER stress reduced cellular susceptibility to bilirubin and apoptosis, while silencing of PERK pathway reduced IL-8, but not TNF-a.

The connection between oxidative stress and inflammation was shown in studies with $\mathrm{Tg}\left(\mathrm{Ugt}^{\mathrm{A} 1^{*} 28}\right) \mathrm{Ugt}^{-/-} / \mathrm{TIr} 2^{-/-}$and $\mathrm{Ugt}^{-1-} \mathrm{FVB} / \mathrm{NJ}$ animals. ${ }^{52,74}$ Both microglia and astrocytes are significantly increased in immunoreactive brain sections derived from $\operatorname{Tg}\left(\mathrm{Ugt}^{\mathrm{A} 1 * 28}\right) \mathrm{Ugt}^{-/-}$showing kernicterus. ${ }^{52}$ When TLR2 (the only TLR isoform stimulated by hyperbilirubinemia) is absent, $\operatorname{Tg}\left(\mathrm{Ugt}^{\mathrm{A} 1^{*} 28}\right) \mathrm{Ugt} 1^{-1-} / \mathrm{Tlr} 2^{-/-}$mice fail to activate inflammatory response and glia reactivity, but die earlier than $\mathrm{Tg}\left(\mathrm{Ugt}^{\mathrm{A} 1^{*} 28}\right) \mathrm{Ugt}^{-/-}$controls. $^{52}$ Pro-inflammatory M1 microglia is increasingly activated over time in Ugt $1^{-1-} \mathrm{FVB} / \mathrm{NJ}$ mice, while anti-inflammatory $\mathrm{M} 2$ microglia is activated during the early stages and decreases as neurotoxicity intensifies. ${ }^{74}$ Treatment with minocycline, a well-known anti-inflammatory tetracycline, results in a dose-dependent rescue of neonatal lethality, accompanied by inhibition of neurotoxicity and neuroinflammation. ${ }^{75}$ Interestingly, ER stress response is only partially attenuated and CHOP reactivity is localized in M1 microglia cells in minocycline-treated mice, suggesting that oxidative stress may not be the primary cause of neurotoxicity and other pathways may be involved.

In vitro studies showed that toxic levels of bilirubin activate autophagy in HBMEC and SH-SY5Y, but not in astrocytes, ${ }^{34,105}$ and autophagy is reduced by the pharmacological inhibition of either ER stress or calcium release, indicating its direct association with ER stress response and calcium signaling. ${ }^{34}$ Conversely, in vivo autophagy is increased only during the latest phases of neurodegeneration and neuroinflammation. ${ }^{74}$
Bilirubin accumulation in the brain and transporters

In normal conditions $\mathrm{Bf}$ levels are negligible, accounting for $<0.1 \%$ of total bilirubin, ${ }^{106}$ but during unconjugated hyperbilirubinemia, the bilirubin-binding capacity by albumin is exceeded and $\mathrm{Bf}$ passes the blood-brain barrier producing neurological damage. ${ }^{107}$ The increase in bilirubin-binding capacity by albumin administration has been performed in subjects prior to exchange transfusion for more than 50 years. ${ }^{108}$ However, the therapeutic use has not been adopted due to the lack of strong experimental support. The interest reawakened in the recent years due to its application to animal models. ${ }^{76,83,84,109}$ These studies show that albumin infusion prior to $\mathrm{PHZ}$ and sulfadimetoxine challenge significantly improve the outcome of Gunn rats. ${ }^{80,83,84}$ Likewise, daily albumin administration to Ugt $1^{-/-} \mathrm{FVB} / \mathrm{NJ}$ pups prevents bilirubin accumulation in the brain, neurological damage, and death. $^{76}$

Another important field of investigation regards the modulation of bilirubin extrusion from the brain by stimulating active transporters. An abundant literature explored the contribution of different $A B C$ transporters to bilirubin detoxification in vitro and in vivo. ${ }^{27,28,41,42,110-112}$ Among them, $A B C B 1$ and $A B C C 1$ appeared to be the main ones limiting bilirubin accumulation in the CNS. $^{81,112,113}$ The key role of Abcb1 was demonstrated using double and triple $\mathrm{KO}$ mice deficient in bilirubin glucuronidation and with null mutations in the $A b c b 1$ and $A b c c 1$ genes. ${ }^{36}$ Results showed that $A b c b 1$, but not $A b c c 1$, improves survival of mutant mice, ${ }^{36}$ underscoring the importance of this transporter in limiting bilirubin accumulation in the brain. In line with these results, $A b c b 1$ gene expression is upregulated in the cerebellum of hypebilirubinemic pups, ${ }^{36}$ but is lower in $\operatorname{Tg}\left(\mathrm{Ugt}^{\mathrm{A1}{ }^{* 28}}\right)$ Ugt $1^{-1-} / \mathrm{CAR}^{-1-}$ and animals die from kernicterus, suggesting their inability to efficiently clear bilirubin from the brain. ${ }^{12}$

\section{Modulation of enzymatic pathways}

Bilirubin formation and its elimination are the result of a sequence of metabolic reactions. ${ }^{114}$ Thus, different strategies to limit UCB production or to increase its degradation have been explored.

The blockade of biliverdin production can be obtained with metalloporphyrins, which are heme byproducts blocking HO-1 activity. ${ }^{115}$ Zinc protoporphyrin administered to Ugt $1^{-/-}$pups reduces bilirubin levels rescuing lethality in a dose-dependent manner. ${ }^{116}$ Despite their proved efficacy in animal models and in clinical trials, ${ }^{117,118}$ their clinical use is still avoided due to several side effects, such as anemia, photosensitivity, lipid peroxidation, increase in $\mathrm{HO}-1$ expression, and reduction of Cyp450 activity. ${ }^{19-122}$

Thus, alternative approaches are currently under investigation, such as blocking the transition from biliverdin to bilirubin. Biliverdin is a non-toxic water-soluble compound that can be easily eliminated in bile and urine. ${ }^{123,124}$ Biliverdin reductase (BLVRA) is not present, or have reduced activity, in different species such as some fishes and the majority of reptiles and birds, in which biliverdin is eliminated through their feces. ${ }^{125}$ It is hypothesized that conversion of biliverdin into bilirubin is required to allow elimination of heme catabolites from the fetus in placental mammals. ${ }^{123,126}$ Interestingly, humans lacking the BLVRA enzyme due to mutations are asymptomatic, indicating that hyperbiliverdinemia is harmless. ${ }^{127,128}$ Screening of a library of Food and Drug Administration-approved compounds identified those able to block biliverdin conversion in tissue culture cells. However, in vivo testing resulted in negative results due to toxicity or lack of efficacy. ${ }^{129}$ The evidence that patients ${ }^{127,128}$ and mice ${ }^{130}$ lacking BLVRA activity are normal strongly support further studies to determine the therapeutic applicability of this approach in acute severe hyperbilirubinemia conditions.

Finally, another approach to reduce hyperbilirubinemia is to stimulate alternative pathways of bilirubin detoxification, such as 
oxidation throughout the activity of cytochromes CYP2A6, CYP1A1, and CYP1A1A2. ${ }^{131,132} \beta$-Naphtoflavone, a potent inducer of Cyp1a1/a2, was tested in Ugt $1^{-1-}$ neonatal mice resulting in extended survival. ${ }^{116}$ We can conclude that, although very preliminary, the results obtained so far are very promising and require further investigation.

The traditional Chinese medicine has used a variety of herbs to lower bilirubin levels in neonatal jaundice. ${ }^{133-136}$ An important study from Huang et al. ${ }^{137}$ demonstrated that Yin Chin and 6,7dimethylesculetin, two components of Yin Zhi Huang, induce CAR target gene expression and improve bilirubin clearance ${ }^{137}$ mediated by the increased translocation of CAR from the cytoplasm to the nucleus, rather than an upregulation of its gene expression. Importantly, among the downstream genes affected by CAR induction, Ugt1a1 and Cyp2b10 were the most stimulated ones.

Overall, results obtained so far point to CAR as a key transcription factor improving bilirubin clearance. To this respect, testing drugs targeting specific CAR-induced genes in Ugt1-null mice will be crucial to elucidate which genes can increase UCB detoxification in the absence of Ugt1a1 enzyme.

Hepatic and gastrointestinal factors contributing to neonatal hyperbilirubinemia

Besides genetic-based factors, other aspects affect bilirubin levels during the neonatal period. Recent studies reported that PXR tightly regulates Ugt1 expression during perinatal period in both the liver and gastrointestinal tract. ${ }^{12,63}$ In fact, Ugt1a1 hepatic expression in neonates does not correlate with bilirubin levels, while gastrointestinal Ugt1a1 expression is present as early as 5 days after birth. ${ }^{63}$ Thereafter, studies using PXR ${ }^{-1-}$ animals mated with $\mathrm{Tg}\left(\mathrm{Ugt} 1^{\mathrm{A} 1^{*} 28}\right) \mathrm{Ugt1^{-/- }}$ mice showed that, in the absence of PXR, pups express more Ugt1a1 enzyme in the liver and have lower TB levels, ${ }^{138}$ demonstrating that PXR is a transcriptional repressor of hepatic Ugt1a1 in the neonatal period, but not in the adult. Further studies showed the participation of NCoR1 (nuclear receptor co-repressor 1) in Ugt1a transcriptional regulation during development. ${ }^{139}$ Thus, the identification of nuclear receptors as key regulators of hepatic Ugt1a1 expression during the neonatal period may lead to the development of alternative approaches to influence bilirubin clearance in neonates.

Conversely, breeding of $\operatorname{Tg}\left(\mathrm{UGT}_{1}^{\mathrm{A} 1^{*} 28}\right) \mathrm{Ugt} 1^{-/-}$with $\mathrm{CAR}^{-/-}$ mice did not affect UGT1A1 expression, indicating a marginal role of CAR in controlling UGT1A1 expression during the neonatal period. To note, modulators of CAR, such as phenobarbital (PhB), have been used to lower bilirubin levels in animal models and is a current treatment for CNI and II patients, together with PT. ${ }^{12,140,141}$ The effects are mediated by the PhB-responsive enhancer module present in the UGT1A1 gene promoter, which is regulated by CAR in response to $\mathrm{PhB}$ treatment. ${ }^{142}$

Breast-fed newborns have more chances to develop neonatal jaundice compared to formula milk fed babies. ${ }^{11,143,144}$ The study of $\mathrm{Tg}\left(\mathrm{Ugt}^{\mathrm{A} 1^{*} 28}\right) \mathrm{Ugt}^{-/-}$mice contributed to understand the molecular mechanisms behind this effect. Breast milk feeding suppresses intestinal IKB kinase $a$ and $b$, resulting in inactivation of nuclear factor-KB and loss of intestine UGT1A1 expression, and high bilirubin levels. ${ }^{12}$ In contrast, newborns treated with formula milk show significantly lower plasma bilirubin levels, correlating with a dramatic increase in intestinal Ugt1a1a expression (200-300-fold). Furthermore, reduction in bilirubin levels by $\mathrm{PhB}$ is not mediated by CAR, but by Ugt1a1 and Cyp2b10 induction in the intestine through other molecular pathways.

Importantly, breast feeding has well-recognized benefits to the neonate. Thus, strategies to avoid breast milk-mediated Ugt1a1 intestinal suppression are under investigations. Glucose supplementation to breast-feeding mouse neonates increases bilirubin metabolism in the intestine, but not in the liver, reducing plasma bilirubin levels, ${ }^{65}$ supporting the potential application of this simple and very convenient treatment to ameliorate transient neonatal hyperbilirubinemia in neonates.

\section{OPEN QUESTIONS}

The work performed so far in cells and animal models allowed the description of the complex cascade of molecular and cellular events, leading to bilirubin-induced neurotoxicity and kernicterus. While much information can be obtained from cell-based approaches, at present the mouse is the experimental tool of choice for in vivo studies due to the possibility to manipulate their genome and to determine the role of other genes and tissues.

These models are necessary to develop and test new therapeutic strategies aiming at reducing neurological damage by uncontrolled hyperbilirubinemia, at the reach of all neonatal care units and clinical centers.

In effect, recent in vivo experiments showed that high Bf levels in the brain trigger neurodegeneration and neuroinflammation, associated with reduced myelination of neurons, which in turn increase ER stress and oxidative stress.

Very promising therapeutic approaches have been proposed using engineered mice, such as anti-inflammatory therapies, transcriptional activation, albumin infusion, administration of protoporphyrins, or blockade of BLVRA. Other promising potential approaches regard the modulation of nuclear receptors, cytochromes or biliverdin reductase activity, in order to control bilirubin production or to stimulate alternative bilirubin-disposal pathways. However, further studies are still needed to potentially translate these approaches to the clinic. The complete understanding of the molecular mechanisms leading to bilirubin neurotoxicity is of central importance to shed light to human biology and health.

\section{ACKNOWLEDGEMENTS}

This work was supported by ICGEB intramural funds.

\section{AUTHOR CONTRIBUTIONS}

G.B. and A.F.M. wrote, revised and approved the manuscript.

\section{ADDITIONAL INFORMATION}

Competing interests: The authors declare no competing interests.

Publisher's note Springer Nature remains neutral with regard to jurisdictional claims in published maps and institutional affiliations.

\section{REFERENCES}

1. Seppen, J. et al. Discrimination between Crigler-Najjar type I and II by expression of mutant bilirubin uridine diphosphate-glucuronosyltransferase. J. Clin. Investig. 94, 2385-2391 (1994).

2. Memon, N., Weinberger, B. I., Hegyi, T. \& Aleksunes, L. M. Inherited disorders of bilirubin clearance. Pediatr. Res. 79, 378-386 (2016).

3. American Academy of Pediatrics Subcommittee on $\mathrm{H}$. Management of hyperbilirubinemia in the newborn infant 35 or more weeks of gestation. Pediatrics 114, 297-316 (2004).

4. Ullah, S., Rahman, K. \& Hedayati, M. Hyperbilirubinemia in neonates: types, causes, clinical examinations, preventive measures and treatments: a narrative review article. Iran. J. Public Health 45, 558-568 (2016).

5. Sedlak, T. W. \& Snyder, S. H. Bilirubin benefits: cellular protection by a biliverdin reductase antioxidant cycle. Pediatrics 113, 1776-1782 (2004).

6. Stocker, R. Antioxidant activities of bile pigments. Antioxid. Redox Signal. 6, 841-849 (2004).

7. Dani, C., Poggi, C. \& Pratesi, S. Bilirubin and oxidative stress in term and preterm infants. Free Radical Res. 53, 2-7 (2019).

8. Coughtrie, M. W., Burchell, B., Leakey, J. E. \& Hume, R. The inadequacy of perinatal glucuronidation: immunoblot analysis of the developmental expression of 
individual UDP-glucuronosyltransferase isoenzymes in rat and human liver microsomes. Mol. Pharmacol. 34, 729-735 (1988).

9. Watchko, J. F. \& Tiribelli, C. Bilirubin-induced neurologic damage-mechanisms and management approaches. N. Engl. J. Med. 369, 2021-2030 (2013).

10. Watchko, J. F. In Avery's Diseases of the Newborn 10th edn (eds Gleason, C. \& Juul, S.) (Elsevier, Philadelphia, 2018).

11. Arias, I. M., Gartner, L. M., Seifter, S. \& Furman, M. Prolonged neonatal unconjugated hyperbilirubinemia associated with breast feeding and a steroid, pregnane-3(alpha), 20(beta)-diol, in maternal milk that inhibits glucuronide formation in vitro. J. Clin. Invest. 43, 2037-2047 (1964).

12. Fujiwara, R., Chen, S., Karin, M. \& Tukey, R. H. Reduced expression of UGT1A1 in intestines of humanized UGT1 mice via inactivation of NF-kappaB leads to hyperbilirubinemia. Gastroenterology 142, 109-18 e2 (2012).

13. Maisels, M. J. \& McDonagh, A. F. Phototherapy for neonatal jaundice. N. Engl. J. Med. 358, 920-928 (2008).

14. Bhutani, V. K. \& Wong, R. J. Bilirubin neurotoxicity in preterm infants: risk and prevention. J. Clin. Neonatol. 2, 61-69 (2013).

15. Ibekwe, R. C., Ibekwe, M. U. \& Muoneke, V. U. Outcome of exchange blood transfusions done for neonatal jaundice in abakaliki, South eastern Nigeria. $J$. Clin. Neonatol. 1, 34-37 (2012).

16. Hosseinpour Sakha, S. \& Gharehbaghi, M. M. Exchange transfusion in severe hyperbilirubinemia: an experience in northwest Iran. Turkish J. Pediatr. 52, 367-371 (2010).

17. Jackson, J. C. Adverse events associated with exchange transfusion in healthy and ill newborns. Pediatrics 99, E7 (1997).

18. Keenan, W. J., Novak, K. K., Sutherland, J. M., Bryla, D. A. \& Fetterly, K. L. Morbidity and mortality associated with exchange transfusion. Pediatrics 75, 417-421 (1985).

19. Sa, C. A. M., Santos, M. C. P., de Carvalho, M. \& Moreira, M. E. L. Adverse events related to exchange transfusion in newborn infants with hemolytic disease: ten years of experience. Rev. Paul. Pediatr. 27, 168-172 (2009).

20. Davutoglu, M., Garipardic, M., Guler, E., Karabiber, H. \& Erhan, D. The etiology of severe neonatal hyperbilirubinemia and complications of exchange transfusion. Turkish J. Pediatr. 52, 163-166 (2010).

21. Calligaris, R. et al. A transcriptome analysis identifies molecular effectors of unconjugated bilirubin in human neuroblastoma SH-SY5Y cells. BMC Genomics 10, 543 (2009).

22. Qaisiya, M. et al. Bilirubin-induced ER stress contributes to the inflammatory response and apoptosis in neuronal cells. Arch. Toxicol. 91, 1847-1858 (2017).

23. Rawat, V., Bortolussi, G., Gazzin, S., Tiribelli, C. \& Muro, A. F. Bilirubin-induced oxidative stress leads to DNA damage in the cerebellum of hyperbilirubinemic neonatal mice and activates DNA double-strand break repair pathways in human cells. Oxid. Med. Cell. Longev. 2018, 1801243 (2018).

24. Cesaratto, L. et al. Bilirubin-induced cell toxicity involves PTEN activation through an APE1/Ref-1-dependent pathway. J. Mol. Med. 85, 1099-1112 (2007).

25. Deganuto, M. et al. A proteomic approach to the bilirubin-induced toxicity in neuronal cells reveals a protective function of DJ-1 protein. Proteomics 10, 1645-1657 (2010).

26. Oakes, G. H. \& Bend, J. R. Global changes in gene regulation demonstrate that unconjugated bilirubin is able to upregulate and activate select components of the endoplasmic reticulum stress response pathway. J. Biochem. Mol. Toxicol. 24, 73-88 (2010).

27. Calligaris, $\mathrm{S}$. et al. Multidrug resistance associated protein 1 protects against bilirubin-induced cytotoxicity. FEBS Lett. 580, 1355-1359 (2006).

28. Rigato, I., Pascolo, L., Fernetti, C., Ostrow, J. D. \& Tiribelli, C. The human multidrug-resistance-associated protein MRP1 mediates ATP-dependent transport of unconjugated bilirubin. Biochem. J. 383, 335-341 (2004).

29. Zhang, B., Yang, X. \& Gao, X. Taurine protects against bilirubin-induced neurotoxicity in vitro. Brain Res. 1320, 159-167 (2010).

30. Vaz, A. R. et al. Selective vulnerability of rat brain regions to unconjugated bilirubin. Mol. Cell. Neurosci. 48, 82-93 (2011).

31. Calligaris, S. D. et al. Cytotoxicity is predicted by unbound and not total bilirubin concentration. Pediatr. Res. 62, 576-580 (2007).

32. Oakes, G. H. \& Bend, J. R. Early steps in bilirubin-mediated apoptosis in murine hepatoma (Hepa 1c1c7) cells are characterized by aryl hydrocarbon receptorindependent oxidative stress and activation of the mitochondrial pathway. J. Biochem. Mol. Toxicol. 19, 244-255 (2005).

33. Seubert, J. M., Darmon, A. J., El-Kadi, A. O., D'Souza, S. J. \& Bend, J. R. Apoptosis in murine hepatoma hepa 1c1c7 wild-type, $\mathrm{C} 12$, and $\mathrm{C} 4$ cells mediated by bilirubin. Mol. Pharmacol. 62, 257-264 (2002)

34. Qaisiya, M., Mardesic, P., Pastore, B., Tiribelli, C. \& Bellarosa, C. The activation of autophagy protects neurons and astrocytes against bilirubin-induced cytotoxicity. Neurosci. Lett. 661, 96-103 (2017).

35. Brites, D. The evolving landscape of neurotoxicity by unconjugated bilirubin: role of glial cells and inflammation. Front. Pharmacol. 3, 88 (2012).
36. Bockor, L. et al. Modulation of bilirubin neurotoxicity by the Abcb1 transporter in the Ugt1-/- lethal mouse model of neonatal hyperbilirubinemia. Hum. Mol. Genet. 26, 145-157 (2017).

37. Rodrigues, C. M., Sola, S., Silva, R. F. \& Brites, D. Aging confers different sensitivity to the neurotoxic properties of unconjugated bilirubin. Pediatr. Res. 51, 112-118 (2002).

38. Gazzin, S. et al. Bilirubin accumulation and Cyp mRNA expression in selected brain regions of jaundiced Gunn rat pups. Pediatr. Res. 71, 653-660 (2012).

39. Barateiro, A. et al. Reduced myelination and increased glia reactivity resulting from severe neonatal hyperbilirubinemia. Mol. Pharmacol. 89, 84-93 (2016).

40. Brito, M. A., Pereira, P., Barroso, C., Aronica, E. \& Brites, D. New autopsy findings in different brain regions of a preterm neonate with kernicterus: neurovascular alterations and up-regulation of efflux transporters. Pediatr. Neurol. 49, 431-438 (2013).

41. Bellarosa, C., Bortolussi, G. \& Tiribelli, C. The role of ABC transporters in protecting cells from bilirubin toxicity. Curr. Pharm. Des. 15, 2884-2892 (2009).

42. Ostrow, J. D., Pascolo, L., Brites, D. \& Tiribelli, C. Molecular basis of bilirubininduced neurotoxicity. Trends Mol. Med. 10, 65-70 (2004).

43. Dal Ben, M., Bottin, C., Zanconati, F., Tiribelli, C. \& Gazzin, S. Evaluation of region selective bilirubin-induced brain damage as a basis for a pharmacological treatment. Sci. Rep. 7, 41032 (2017).

44. Brites, D. \& Fernandes, A. Bilirubin-induced neural impairment: a special focus on myelination, age-related windows of susceptibility and associated comorbidities. Semin. Fetal Neonatal Med. 20, 14-19 (2015).

45. Falcao, A. S. et al. Cross-talk between neurons and astrocytes in response to bilirubin: early beneficial effects. Neurochem. Res. 38, 644-659 (2013).

46. Silva, S. L. et al. Neuritic growth impairment and cell death by unconjugated bilirubin is mediated by NO and glutamate, modulated by microglia, and prevented by glycoursodeoxycholic acid and interleukin-10. Neuropharmacology 62, 2398-2408 (2012).

47. Chang, F. Y., Lee, C. C., Huang, C. C. \& Hsu, K. S. Unconjugated bilirubin exposure impairs hippocampal long-term synaptic plasticity. PLOS ONE 4, e5876 (2009).

48. Dani, C. et al. Neurotoxicity of unconjugated bilirubin in mature and immature rat organotypic hippocampal slice cultures. Neonatology 115, 217-225 (2019).

49. Silva, S. L. et al. Features of bilirubin-induced reactive microglia: from phagocytosis to inflammation. Neurobiol. Dis. 40, 663-675 (2010).

50. Bortolussi, G. et al. Age-dependent pattern of cerebellar susceptibility to bilirubin neurotoxicity in vivo in mice. Dis. Model. Mech. 7, 1057-1068 (2014).

51. Bortolussi, G. et al. Rescue of bilirubin-induced neonatal lethality in a mouse model of Crigler-Najjar syndrome type I by AAV9-mediated gene transfer. FASEB J. 26, 1052-1063 (2012).

52. Yueh, M. F., Chen, S., Nguyen, N. \& Tukey, R. H. Developmental onset of bilirubininduced neurotoxicity involves Toll-like receptor 2-dependent signaling in humanized UDP-glucuronosyltransferase1 mice. J. Biol. Chem. 289, 4699-4709 (2014).

53. Schutta, H. S. \& Johnson, L. Bilirubin encephalopathy in the Gunn rat: a fine structure study of the cerebellar cortex. J. Neuropathol. Exp. Neurol. 26, 377-396 (1967).

54. Shapiro, S. M. Bilirubin toxicity in the developing nervous system. Pediatr. Neurol. 29, 410-421 (2003).

55. Shapiro, S. M. Acute brainstem auditory evoked potential abnormalities in jaundiced Gunn rats given sulfonamide. Pediatr. Res. 23, 306-310 (1988).

56. Conlee, J. W. \& Shapiro, S. M. Development of cerebellar hypoplasia in jaundiced Gunn rats: a quantitative light microscopic analysis. Acta Neuropathol. 93, 450-460 (1997).

57. Gunn, C. K. Hereditary acholuric jaundice in the rat. Can. Med. Assoc. J. $\mathbf{5 0}$ 230-237 (1944).

58. Gunn, C. K. Hereditary acholuric jaundice in a nre mutant strain of rats. J. Hered. 29, 137-139 (1938)

59. Iyanagi, T., Watanabe, T. \& Uchiyama, Y. The 3-methylcholanthrene-inducible UDP-glucuronosyltransferase deficiency in the hyperbilirubinemic rat (Gunn rat) is caused by a -1 frameshift mutation. J. Biol. Chem. 264, 21302-21307 (1989).

60. Emi, Y., Omura, S., Ikushiro, S. \& lyanagi, T. Accelerated degradation of mislocalized UDP-glucuronosyltransferase family 1 (UGT1) proteins in Gunn rat hepatocytes. Arch. Biochem. Biophys. 405, 163-169 (2002).

61. Sawasaki, Y., Yamada, N. \& Nakajima, H. Developmental features of cerebellar hypoplasia and brain bilirubin levels in a mutant (Gunn) rat with hereditary hyperbilirubinaemia. J. Neurochem. 27, 577-583 (1976).

62. Chen, S. et al. Intestinal glucuronidation protects against chemotherapyinduced toxicity by irinotecan (CPT-11). Proc. Natl Acad. Sci. USA 110, 19143-19148 (2013).

63. Fujiwara, R., Nguyen, N., Chen, S. \& Tukey, R. H. Developmental hyperbilirubinemia and CNS toxicity in mice humanized with the UDP glucuronosyltransferase 1 (UGT1) locus. Proc. Natl Acad. Sci. USA 107, 5024-5029 (2010). 
64. Nguyen, N. et al. Disruption of the ugt1 locus in mice resembles human Crigler-Najjar type I disease. J. Biol. Chem. 283, 7901-7911 (2008).

65. Aoshima, N., Fujie, Y., Itoh, T., Tukey, R. H. \& Fujiwara, R. Glucose induces intestinal human UDP-glucuronosyltransferase (UGT) 1A1 to prevent neonatal hyperbilirubinemia. Sci. Rep. 4, 6343 (2014).

66. Sumida, K. et al. Importance of UDP-glucuronosyltransferase $1 \mathrm{~A} 1$ expression in skin and its induction by UVB in neonatal hyperbilirubinemia. Mol. Pharmacol. 84, 679-686 (2013)

67. Fujiwara, R., Maruo, Y., Chen, S. \& Tukey, R. H. Role of extrahepatic UDPglucuronosyltransferase 1A1: advances in understanding breast milk-induced neonatal hyperbilirubinemia. Toxicol. Appl. Pharmacol. 289, 124-132 (2015).

68. Bockor, L. et al. Repeated AAV-mediated gene transfer by serotype switching enables long-lasting therapeutic levels of hUgt1a1 enzyme in a mouse model of Crigler-Najjar syndrome type I. Gene Ther. 24, 649-660 (2017).

69. Bortolussi, G. \& Muro, A. F. Advances in understanding disease mechanisms and potential treatments for Crigler-Najjar syndrome. Expert Opin. Orphan Drugs 6, 425-439 (2018)

70. Bortolussi, G. et al. Life-long correction of hyperbilirubinemia with a neonatal liver-specific AAV-mediated gene transfer in a lethal mouse model of Crigler-Najjar Syndrome. Hum. Gene Ther. 25, 844-855 (2014).

71. Collaud, F. et al. Preclinical development of an AAV8-hUGT1A1 vector for the treatment of Crigler-Najjar Syndrome. Mol. Ther. Methods Clin. Dev. 12, 157-174 (2019).

72. Miranda, P. S. \& Bosma, P. J. Towards liver-directed gene therapy for Crigler-Najjar syndrome. Curr. Gene Ther. 9, 72-82 (2009).

73. Ronzitti, G. et al. A translationally optimized AAV-UGT1A1 vector drives safe and long-lasting correction of Crigler-Najjar syndrome. Mol. Ther. Methods Clin. Dev. 3, 16049 (2016).

74. Vodret, S., Bortolussi, G., Jasprova, J., Vitek, L. \& Muro, A. F. Inflammatory signature of cerebellar neurodegeneration during neonatal hyperbilirubinemia in Ugt1 (-/-) mouse model. J. Neuroinflammation 14, 64 (2017).

75. Vodret, S. et al. Attenuation of neuro-inflammation improves survival and neurodegeneration in a mouse model of severe neonatal hyperbilirubinemia. Brain Behav. Immun. 70, 166-178 (2018).

76. Vodret, S. et al. Albumin administration prevents neurological damage and death in a mouse model of severe neonatal hyperbilirubinemia. Sci. Rep. 5, 16203 (2015)

77. Gao, X., Yang, X. \& Zhang, B. Neuroprotection of taurine against bilirubininduced elevation of apoptosis and intracellular free calcium ion in vivo. Toxicol. Mech. Methods 21, 383-387 (2011).

78. Huang, $\mathrm{H}$. et al. Bilirubin neurotoxicity is associated with proteasome inhibition. Cell Death Dis. 8, e2877 (2017).

79. Song, S., Hu, Y., Gu, X., Si, F. \& Hua, Z. A novel newborn rat kernicterus model created by injecting a bilirubin solution into the cisterna magna. PLOS ONE 9 , e96171 (2014).

80. Rice, A. C. \& Shapiro, S. M. A new animal model of hemolytic hyperbilirubinemia-induced bilirubin encephalopathy (kernicterus). Pediatr. Res. 64, 265-269 (2008).

81. Cekic, D. et al. Upregulation in the expression of multidrug resistance protein Mrp1 mRNA and protein by increased bilirubin production in rat. Biochem. Biophys. Res. Commun. 311, 891-896 (2003)

82. Maity, S., Nag, N., Chatterjee, S., Adhikari, S. \& Mazumder, S. Bilirubin clearance and antioxidant activities of ethanol extract of Phyllanthus amarus root in phenylhydrazine-induced neonatal jaundice in mice. J. Physiol. Biochem. 69, 467-476 (2013).

83. Schreuder, A. B. et al. Albumin administration protects against bilirubin-induced auditory brainstem dysfunction in Gunn rat pups. Liver Int. 33, 1557-1565 (2013).

84. Schreuder, A. B. et al. Optimizing exchange transfusion for severe unconjugated hyperbilirubinemia: studies in the Gunn rat. PLOS ONE 8, e77179 (2013).

85. Davies, B. E. Displacement of bilirubin from cord serum by sulphadimethoxine, amoxycillin, clavulanic acid in combination with either amoxycillin or ticarcillin, temocillin and cloxacillin. Br. J. Clin. Pharmacol. 20, 345-348 (1985)

86. Diamond, I. Bilirubin encephalopathy: experimental models in newborn and adult animals. Proc. Inst. Med. Chic. 26, 47-48 (1966).

87. Davis, D. R. \& Yeary, R. A. Effects of sulfadimethoxine on tissue distribution of (14C)bilirubin in the newborn and adult hyperbilirubinemic Gunn rate. Pediatr. Res. 9, 846-850 (1975).

88. Shapiro, S. M. \& Conlee, J. W. Brainstem auditory evoked potentials correlate with morphological changes in Gunn rat pups. Hearing Res. 57, 16-22 (1991).

89. Shapiro, S. M. Somatosensory and brainstem auditory evoked potentials in the Gunn rat model of acute bilirubin neurotoxicity. Pediatr. Res. 52, 844-849 (2002).

90. Daood, M. J. \& Watchko, J. F. Calculated in vivo free bilirubin levels in the central nervous system of Gunn rat pups. Pediatr. Res. 60, 44-49 (2006).
91. Keino, H. \& Kashiwamata, S. Critical period of bilirubin-induced cerebellar hypoplasia in a new Sprague-Dawley strain of jaundiced Gunn rats. Neurosci. Res. 6, 209-215 (1989).

92. Tell, G. \& Gustincich, S. Redox state, oxidative stress, and molecular mechanisms of protective and toxic effects of bilirubin on cells. Curr. Pharm. Des. 15, 2908-2914 (2009).

93. Qaisiya, M., Coda Zabetta, C. D., Bellarosa, C. \& Tiribelli, C. Bilirubin mediated oxidative stress involves antioxidant response activation via Nrf2 pathway. Cell Signal. 26, 512-520 (2014).

94. Bortolussi, G. et al. Impairment of enzymatic antioxidant defenses is associated with bilirubin-induced neuronal cell death in the cerebellum of Ugt1 KO mice. Cell Death Dis. 6, e1739 (2015).

95. Yoda, E. et al. Isothiocyanates induce UGT1A1 in humanized UGT1 mice in a CAR dependent fashion that is highly dependent upon oxidative stress. Sci. Rep. 7, 46489 (2017).

96. Daood, M. J., Hoyson, M. \& Watchko, J. F. Lipid peroxidation is not the primary mechanism of bilirubin-induced neurologic dysfunction in jaundiced Gunn rat pups. Pediatr. Res. 72, 455-459 (2012).

97. Wallner, M. et al. Anti-genotoxic potential of bilirubin in vivo: damage to DNA in hyperbilirubinemic human and animal models. Cancer Prev. Res. 6, 1056-1063 (2013).

98. Vianello, E. et al. Histone acetylation as a new mechanism for bilirubin-induced encephalopathy in the Gunn rat. Sci. Rep. 8, 13690 (2018).

99. Brito, M. A. et al. Cerebellar axon/myelin loss, angiogenic sprouting, and neuronal increase of vascular endothelial growth factor in a preterm infant with kernicterus. J. Child Neurol. 27, 615-624 (2012).

100. Barateiro, A., Vaz, A. R., Silva, S. L., Fernandes, A. \& Brites, D. ER stress, mitochondrial dysfunction and calpain/JNK activation are involved in oligodendrocyte precursor cell death by unconjugated bilirubin. Neuromol. Med. 14, 285-302 (2012).

101. Ding, W. X. et al. Linking of autophagy to ubiquitin-proteasome system is important for the regulation of endoplasmic reticulum stress and cell viability. Am. J. Pathol. 171, 513-524 (2007).

102. Fernandes, A., Silva, R. F., Falcao, A. S., Brito, M. A. \& Brites, D. Cytokine production, glutamate release and cell death in rat cultured astrocytes treated with unconjugated bilirubin and LPS. J. Neuroimmunol. 153, 64-75 (2004).

103. Fernandes, A. et al. Inflammatory signalling pathways involved in astroglial activation by unconjugated bilirubin. J. Neurochem. 96, 1667-1679 (2006).

104. Gordo, A. C. et al. Unconjugated bilirubin activates and damages microglia. J. Neurosci. Res. 84, 194-201 (2006).

105. Palmela, I. et al. Time-dependent dual effects of high levels of unconjugated bilirubin on the human blood-brain barrier lining. Front. Cell. Neurosci. 6, 22 (2012).

106. Brodersen, R. Bilirubin. Solubility and interaction with albumin and phospholipid. J. Biol. Chem. 254, 2364-2369 (1979).

107. Wennberg, R. P., Ahlfors, C. E., Bhutani, V. K., Johnson, L. H. \& Shapiro, S. M. Toward understanding kernicterus: a challenge to improve the management of jaundiced newborns. Pediatrics 117, 474-485 (2006).

108. Odell, G. B., Cohen, S. N. \& Gordes, E. H. Administration of albumin in the management of hyperbilirubinemia by exchange transfusions. Pediatrics $\mathbf{3 0}$, 613-621 (1962).

109. Cuperus, F. J. et al. Beyond plasma bilirubin: the effects of phototherapy and albumin on brain bilirubin levels in Gunn rats. J. Hepatol. 58, 134-140 (2013).

110. Gennuso, F. et al. Bilirubin protects astrocytes from its own toxicity by inducing up-regulation and translocation of multidrug resistance-associated protein 1 (Mrp1). Proc. Natl Acad. Sci. USA 101, 2470-2475 (2004).

111. Falcao, A. S. et al. Role of multidrug resistance-associated protein 1 expression in the in vitro susceptibility of rat nerve cell to unconjugated bilirubin. Neuroscience 144, 878-888 (2007).

112. Watchko, J. F., Daood, M. J. \& Hansen, T. W. Brain bilirubin content is increased in P-glycoprotein-deficient transgenic null mutant mice. Pediatr. Res. 44, 763-766 (1998).

113. Hanko, E., Tommarello, S., Watchko, J. F. \& Hansen, T. W. Administration of drugs known to inhibit P-glycoprotein increases brain bilirubin and alters the regional distribution of bilirubin in rat brain. Pediatr. Res. 54, 441-445 (2003).

114. Tukey, R. H. \& Strassburg, C. P. Human UDP-glucuronosyltransferases: metabolism, expression, and disease. Annu. Rev. Pharm. Toxicol. 40, 581-616 (2000)

115. Drummond, G. S. \& Kappas, A. Sn-protoporphyrin inhibition of fetal and neonatal brain heme oxygenase. Transplacental passage of the metalloporphyrin and prenatal suppression of hyperbilirubinemia in the newborn animal. J. Clin. Invest. 77, 971-976 (1986).

116. Fujiwara, R., Mitsugi, R., Uemura, A., Itoh, T. \& Tukey, R. H. Severe neonatal hyperbilirubinemia in Crigler-Najjar syndrome model mice can be reversed with zinc protoporphyrin. Hepatol. Commun. 1, 792-802 (2017). 
117. Bhutani, V. K. et al. Clinical trial of tin mesoporphyrin to prevent neonatal hyperbilirubinemia. J. Perinatol. 36, 533-539 (2016).

118. Wong, R. J. et al. Effects of light on metalloporphyrin-treated newborn mice. Acta Paediatr. 103, 474-479 (2014).

119. Stevenson, D. K. \& Wong, R. J. Metalloporphyrins in the management of neonatal hyperbilirubinemia. Semin. Fetal Neonatal Med. 15, 164-168 (2010).

120. Schulz, S., Wong, R. J., Vreman, H. J. \& Stevenson, D. K. Metalloporphyrins-an update. Front. Pharmacol. 3, 68 (2012).

121. Keino, H., Nagae, H., Mimura, S., Watanabe, K. \& Kashiwamata, S. Dangerous effects of tin-protoporphyrin plus photoirradiation on neonatal rats. Eur. J. Pediatr. 149, 278-279 (1990).

122. Dennery, P. A., Vreman, H. J., Rodgers, P. A. \& Stevenson, D. K. Role of lipid peroxidation in metalloporphyrin-mediated phototoxic reactions in neonatal rats. Pediatr. Res. 33, 87-91 (1993).

123. McDonagh, A. F. Turning green to gold. Nat. Struct. Biol. 8, 198-200 (2001).

124. McDonagh, A. F., Palma, L. A. \& Schmid, R. Reduction of biliverdin and placental transfer of bilirubin and biliverdin in the pregnant guinea pig. Biochem. J. 194 273-282 (1981).

125. Cornelius, C. E. Bile pigments in fishes: a review. Vet. Clin. Pathol. 20, 106-115 (1991).

126. McDonagh, A. F. Movement of bilirubin and bilirubin conjugates across the placenta. Pediatrics 119, 1032-1033 (2007). author reply 3.

127. Nytofte, N. S. et al. A homozygous nonsense mutation (c.214C->A) in the biliverdin reductase alpha gene (BLVRA) results in accumulation of biliverdin during episodes of cholestasis. J. Med. Genet. 48, 219-225 (2011).

128. Gafvels, M. et al. A novel mutation in the biliverdin reductase-A gene combined with liver cirrhosis results in hyperbiliverdinaemia (green jaundice). Liver Int. 29, 1116-1124 (2009).

129. van Dijk, R. et al. Biliverdin reductase inhibitors did not improve severe unconjugated hyperbilirubinemia in vivo. Sci. Rep. 7, 1646 (2017).

130. Chen, W. et al. Absence of the biliverdin reductase-a gene is associated with increased endogenous oxidative stress. Free Radic. Biol. Med. 115, 156-165 (2018).

131. Abu-Bakar, A. et al. Metabolism of bilirubin by human cytochrome P450 2 A6. Toxicol. Appl. Pharmacol. 261, 50-58 (2012).
132. Kapitulnik, J. \& Ostrow, J. D. Stimulation of bilirubin catabolism in jaundiced Gunn rats by an induced of microsomal mixed-function monooxygenases. Proc. Natl Acad. Sci. USA 75, 682-685 (1978).

133. Yin, J., Miller, M. \& Wennberg, R. P. Induction of hepatic bilirubin-metabolizing enzymes by the traditional Chinese medicine yin zhi huang. Dev. Pharmacol. Ther. 16, 176-184 (1991).

134. Yin, J., Wennberg, R. P., Xia, Y. C., Liu, J. W. \& Zhou, H. Z. Effect of a traditional Chinese medicine, yin zhi huang, on bilirubin clearance and conjugation. Dev. Pharmacol. Ther. 16, 59-64 (1991).

135. Fok, T. F. Neonatal jaundice-traditional Chinese medicine approach. J. Perinatol. 21(Suppl. 1), S98-S100 (2001). discussion S4-7.

136. Ho, N. K. Traditional Chinese medicine and treatment of neonatal jaundice. Singap. Med. J. 37, 645-651 (1996).

137. Huang, W., Zhang, J. \& Moore, D. D. A traditional herbal medicine enhances bilirubin clearance by activating the nuclear receptor CAR. J. Clin. Investig. 113, 137-143 (2004).

138. Chen, S., Yueh, M. F., Evans, R. M. \& Tukey, R. H. Pregnane-x-receptor controls hepatic glucuronidation during pregnancy and neonatal development in humanized UGT1 mice. Hepatology 56, 658-667 (2012).

139. Chen, S. \& Tukey, R. H. Humanized UGT1 mice, regulation of UGT1A1, and the role of the intestinal tract in neonatal hyperbilirubinemia and breast milkinduced jaundice. Drug Metab. Dispos. 46, 1745-1755 (2018).

140. Crigler, J. F. Jr. \& Gold, N. I. Effect of sodium phenobarbital on bilirubin metabolism in an infant with congenital, nonhemolytic, unconjugated hyperbilirubinemia, and kernicterus. J. Clin. Invest. 48, 42-55 (1969).

141. Cohen, A. N. et al. Effects of phenobarbital on bilirubin metabolism and its response to phototherapy in the jaundiced Gunn rat. Hepatology 5, 310-316 (1985).

142. Sugatani, J. et al. The phenobarbital response enhancer module in the human bilirubin UDP-glucuronosyltransferase UGT1A1 gene and regulation by the nuclear receptor CAR. Hepatology 33, 1232-1238 (2001).

143. Gourley, G. R. Breast-feeding, neonatal jaundice and kernicterus. Semin. Neonatol. 7, 135-141 (2002).

144. Maisels, M. J. \& Newman, T. B. Kernicterus in otherwise healthy, breast-fed term newborns. Pediatrics 96, 730-733 (1995). 